\title{
Diagnostic Accuracy of Computed Tomography in Predicting Primary Aldosteronism Subtype According to Age (Endocrinol Metab 2021;36:401-12, Seung Hun Lee et al.)
}

\author{
Pankaj Singhania \\ Department of Endocrinology and Metabolism, IPGME\&R and SSKM Hospital, Kolkata, India
}

We read with interest the article titled "Diagnostic accuracy of computed tomography in predicting primary aldosteronism subtype according to age" by Seung Hun Lee et al. [1] in the March 2021 edition of your esteemed journal.

The investigators did a superb job of presenting primary aldosteronism data from two tertiary care centers in Korea. The remarkably large number of diagnosed cases of primary aldosteronism ( $n=676)$ lends credence to the findings of the study, which tried to address the highly contentious issue of the accurate diagnosis and localization of primary aldosteronism and its source.

Primary aldosteronism is a recognized treatable cause of secondary and resistant young-onset hypertension. The recognition and screening of this entity have markedly increased, to the point that it is no longer considered a rare condition. Studies have shown the prevalence of primary aldosteronism among patients with hypertension to be $10 \%$ [2]. Primary aldosteronism is an etiology of hypertension for which a definitive cure can be offered to patients. Patients undergoing surgery for accurately localized unilateral aldosterone-secreting adrenal adenoma can be cured of hypertension for life and can become drugfree. For those with bilateral disease, the addition of even a small dose of a mineralocorticoid antagonist can significantly

Received: 9 July 2021, Accepted: 12 July 2021

Corresponding author: Pankaj Singhania

Department of Endocrinology and Metabolism, IPGME\&R and SSKM Hospital, 244 AJC Bose Road, Kolkata 70020, India

Tel: +91-9153003736, E-mail: drpankaj007@hotmail.com reduce the pill burden of hypertension and decrease the longterm morbidity and mortality due to uncontrolled hypertension.

The study has a few points with which we agree and some for which we would like to raise a few questions. First, the importance of hypokalemia in diagnosing primary aldosteronismparticularly unilateral adenoma - and thus avoiding the need for adrenal vein sampling (AVS) is perhaps overrated. Hypokalemia (a primary clue for the diagnosis of primary aldosteronism) is present in only $20 \%$ of cases [3]. Therefore, reliance on hypokalemia in evaluating and lateralizing aldosteronism can be erroneous.

Another point of disagreement is the need for confirmatory tests for diagnosing primary aldosteronism in all patients. It is recognized in guidelines that in unequivocal cases, with hypertension in the clinical setting of spontaneous hypokalemia with a plasma aldosterone concentration $>555 \mathrm{pmol} / \mathrm{L}(>20 \mathrm{ng} / \mathrm{dL})$ and plasma renin activity $<1 \mathrm{ng} / \mathrm{mL} / \mathrm{hr}$ (or plasma renin concentration below the lower limit of the reference range); a diagnosis of primary aldosteronism is confirmed and confirmatory testing is not required [4].

Other than these queries, we fully agree with the investigators that although AVS should be the standard of care in the diagnosis of primary aldosteronism, the availability, cost, and technical
Copyright (C) 2021 Korean Endocrine Society

This is an Open Access article distributed under the terms of the Creative Commons Attribution Non-Commercial License (https://creativecommons.org/ licenses/by-nc/4.0/) which permits unrestricted non-commercial use, distribution, and reproduction in any medium, provided the original work is properly cited. 
expertise are important limitations of the widespread use of AVS. The investigators have very clearly tried to predict and accurately diagnose primary aldosteronism based on computed tomography (CT) scan findings with age-specific cut-offs. This would help bypass AVS while still making an accurate diagnosis. We are particularly excited about this possibility because AVS is available in very limited centers in India. Moreover, AVS also has limitations and may be non-diagnostic even in the most experienced hands. Our experiences with AVS at the tertiary care hospital where we work have also not been very encouraging. We often are left with a non-diagnostic AVS. In such cases, we have to revert to clinical findings, biochemical cutoffs, and CT scan findings to arrive at treatment decisions. We look forward to more data from other parts of the world in this regard to make the diagnosis of primary aldosteronism easier, more accurate, and more cost-effective.

\section{CONFLICTS OF INTEREST}

No potential conflict of interest relevant to this article was reported.

\section{ORCID}

Pankaj Singhania https://orcid.org/0000-0002-9392-3300

\section{REFERENCES}

1. Lee SH, Kim JW, Yoon HK, Koh JM, Shin CS, Kim SW, et al. Diagnostic accuracy of computed tomography in predicting primary aldosteronism subtype according to age. Endocrinol Metab (Seoul) 2021;36:401-12.

2. Piaditis G, Markou A, Papanastasiou L, Androulakis II, Kaltsas G. Progress in aldosteronism: a review of the prevalence of primary aldosteronism in pre-hypertension and hypertension. Eur J Endocrinol 2015;172:R191-203.

3. Rossi GP, Bernini G, Caliumi C, Desideri G, Fabris B, Ferri $\mathrm{C}$, et al. A prospective study of the prevalence of primary aldosteronism in 1,125 hypertensive patients. J Am Coll Cardiol 2006;48:2293-300.

4. Funder JW, Carey RM, Mantero F, Murad MH, Reincke M, Shibata $\mathrm{H}$, et al. The management of primary aldosteronism: case detection, diagnosis, and treatment: an Endocrine Society Clinical Practice Guideline. J Clin Endocrinol Metab 2016;101:1889-916. 